\title{
Existence and Uniqueness of Mild Solutions for Nonlinear Stochastic Impulsive Differential Equation
}

\author{
L. J. Shen ${ }^{1,2}$ and J. T. Sun ${ }^{1}$ \\ ${ }^{1}$ Department of Mathematics, Tongji University, Shanghai 200092, China \\ ${ }^{2}$ Department of Mathematics, Luoyang Normal University, Luoyang 471022, China \\ Correspondence should be addressed to J. T. Sun, sunjt@sh163.net
}

Received 9 May 2011; Accepted 9 October 2011

Academic Editor: Jinhu Lü

Copyright (C) 2011 L. J. Shen and J. T. Sun. This is an open access article distributed under the Creative Commons Attribution License, which permits unrestricted use, distribution, and reproduction in any medium, provided the original work is properly cited.

This paper investigates the existence and uniqueness of mild solutions to the general nonlinear stochastic impulsive differential equations. By using Schaefer's fixed theorem and stochastic analysis technique, we propose sufficient conditions on existence and uniqueness of solution for stochastic differential equations with impulses. An example is also discussed to illustrate the effectiveness of the obtained results.

\section{Introduction}

Impulsive dynamical systems often exhibit continuous evolutions typically described by ordinary differential equations and instantaneous state jumps. Since many evolution processes, optimal control models in economics, stimulated neural networks, frequencymodulated systems, and some motions of missiles or aircrafts are characterized by the impulsive dynamical behavior, the study of impulsive systems is of great importance. Nowadays, there has been increasing interest in the analysis and synthesis of impulsive systems due to their significance both in theory and applications; see [1-4] and the references therein.

On the other hand, stochastic differential equations (SDEs) have attracted a great attention since they have been used extensively in many areas of application including finance and social science. Existence, uniqueness, and qualitative analysis of solutions of SDEs have been considered by many authors; see, for example, [5, 6] and Oksendal [7]. In particular, [7] has obtained sufficient conditions (uniform Lipschitz condition and the linear growth condition) on the existence and uniqueness of solution of SDEs. With the same 
conditions, Mao [8] has also proved there exists a unique solution for neutral stochastic functional differential equations with finite delay, and systems with infinite delay was investigated by [9] under uniform Lipschitz condition and moment estimate.

Furthermore, besides impulsive effects, stochastic effects likewise exist in real systems. Therefore, stochastic impulsive differential equations (SIDEs) describing these dynamical systems subject to both stochastic and impulse changes have attracted considerable attention [10-14]. Liu et al. [10] has proved the existence and uniqueness with Lipschitz and linear growth condition by continuation method. Results of existence and uniqueness were obtained by stochastic Lyapunov-type method in [11,12]. Besides that, the existing literature of SIDEs involved mainly the issue on controllability and stability (see $[13,14]$ ), and little investigation, to our best knowledge, has been carried out for existence and uniqueness of general nonlinear systems with stochastic impulsive structure. Unlike the previous results in [10-12], this paper will deal with the situation when the drift and diffusion coefficients do not satisfy Lipschitz condition. Sufficient conditions of existence and uniqueness of mild solutions for general nonlinear SIDEs are developed based on the Schaefer's fixed point theorem instead of employing stochastic Lyapunov-type function. In addition, some known results can been considered as special cases of the main theorems in this paper.

The organization of this paper is as follows. In Section 1, we will recall briefly some basic notations and preliminary facts which will be used throughout the paper. In Section 2, sufficient conditions are obtained on existence and uniqueness of mild solutions to SIDEs. A numerical example is given to illustrate the proposed conditions in Section 3. Finally, concluding remarks are presented in Section 4.

\section{Preliminaries}

Let $\|\cdot\|$ denote the Euclidean norm in $R^{n}$. If $A$ is a matrix, its Frobenius norm is represented by $\|A\|=\sqrt{\operatorname{tr}\left(A^{T} A\right)} \cdot\{\Omega, \mathcal{F}, \mathbb{P}\}$ stands for a complete probability space with a filtration $\left\{\mathcal{F}_{t}\right\}_{t \geq 0}$ satisfying the usual conditions (i.e., right continuous and $\mathcal{F}_{0}$ containing all $\mathbb{P}$-null sets). Assume that $B(t)=\left(B_{1}(t), B_{2}(t), \ldots, B_{l}(t)\right)^{T}$ is an $l$-dimensional Brownian motion defined on $\{\Omega, \mathcal{F}, \mathbb{P}\}$. Denote $L_{2}^{\mathscr{F}}\left(J, R^{n}\right)$ as the family of all square integrable and $\mathcal{F}_{t}$ adapted measurable processes $x(t)=x(t, w)$ mapping $J=[0, b]$ into $R^{n}$ such that $\sup _{0<s \leq t} \mathbb{E}\|x(s)\|^{2}<\infty$. It is clear that $L_{2}^{\mp}\left(J, R^{n}\right)$ is the Banach space with the norm topology given by $\|x(t)\|_{*}^{2}=$ $\sup _{0 \leq s \leq t} \mathbb{E}\|x(s)\|^{2}$.

Consider stochastic differential equations with impulsive effects as follows:

$$
\begin{gathered}
\mathrm{d} x(t)=f(t, x(t)) \mathrm{d} t+g(t, x(t)) \mathrm{d} B(t), \quad t \in J, t \neq \tau_{k}, \\
\Delta x\left(\tau_{k}^{+}\right)=I_{k}\left(x\left(\tau_{k}\right)\right), \quad k=1,2, \ldots, m, \\
x(0)=z,
\end{gathered}
$$

where $f: J \times R^{n} \rightarrow R^{n}, g: J \times R^{n} \rightarrow R^{n \times l}$ are Borel measurable functions, $\Delta x\left(\tau_{k}^{+}\right)=$ $x\left(\tau_{k}^{+}\right)-x\left(\tau_{k}\right)=x\left(\tau_{k}^{+}\right)-x\left(\tau_{k}^{-}\right), I_{k} \in C\left(R^{n}, R^{n}\right)$. The initial value $z$ is a $\mathcal{F}_{0}$-measurable random variable independent of $B(t)$ such that $E\|z\|^{2}<\infty$. 
Definition 2.1. The functions $f$ and $g$ are said to be $L_{2}$-Carathéodory if

(1) $f(t, x)$ and $g(t, x)$ are measurable in $t$ and continuous in the second variable a.e. $t \in J$,

(2) for each positive constant $q$, there exists a square integrable function $h_{q}: J \rightarrow R_{+}$ such that

$$
\|f(t, u)\| \vee\|g(t, u)\| \leq h_{q}(t), \quad\|u\| \leq q, \quad \text { a.e. } t \in J
$$

Motivated by $[1,7]$, let us start by defining what we mean by a mild solution of (2.1).

Definition 2.2. A $R^{n}$-valued stochastic process $x(t)=x(t, w) \in L_{2}^{q}\left(J, R^{n}\right)$ defined on $J$ is said to be a mild solution of (2.1) with initial value $x(0)=z$ if the following conditions are satisfied

(1) $x(t, w)$ is measurable as a function from $J \times \Omega$ to $R^{n}$ and $x(t)$ is $\mathcal{F}_{t}$-adapted;

(2) $E\|x\|^{2}<\infty$ for each $t \in J$;

(3) for each $z \in L_{2}^{\mp}\left(J, R^{n}\right)$, the process $x$ satisfies the following integral equation:

$$
x(t)=z+\int_{0}^{t} f(s, x(s)) \mathrm{d} s+\int_{0}^{t} g(s, x(s)) \mathrm{d} B(s)+\sum_{0<\tau_{k}<t} I_{k}\left(x\left(\tau_{k}\right)\right)
$$

Our main result is based on the following fixed point theorem of Schaefer.

Lemma 2.3 (Schaefer fixed-point theorem). Let $(C,\|\cdot\|)$ be a convex subset of a normal linear space, and let the operator $A: C \rightarrow C$ be completely continuous. Define

$$
F(A)=\{x \in C: x=\lambda A x, \lambda \in(0,1)\} .
$$

Then, either

(1) the set $F(A)$ is unbounded or

(2) the operator $A$ has a fixed point in $C$.

\section{Main Results}

We are now in a position to state and prove our existence result of (2.1). Throughout this paper, we make the following assumptions.

$\left(H_{1}\right)$ The functions $f: J \times R^{n} \rightarrow R^{n}$ and $g: J \times R^{n} \rightarrow R^{n \times l}$ are $L_{2}$-Carathéodory.

$\left(H_{2}\right)$ There exist positive constants $c_{k}, k=1,2, \ldots, m$, such that

$$
\left\|I_{k}(x)\right\| \leq c_{k}, \quad x \in L_{2}^{\mp}\left(J, R^{n}\right) .
$$

$\left(H_{3}\right)$ There exists a square integrable function $p: J \rightarrow R_{+}$and a continuous nondecreasing function $\psi:[0, \infty) \rightarrow[0, \infty)$ such that

$$
\|f(t, x)\| \vee\|g(t, x)\| \leq p(t) \psi(\|x\|), \quad t \in J, x \in L_{2}^{\mp}\left(J, R^{n}\right),
$$


with

$$
4 \int_{0}^{b}(b+1) p^{2}(s) \mathrm{d} s<\int_{c}^{\infty} \frac{d u}{\psi^{2}(u)}
$$

where $c=4 E\|z\|^{2}$.

Theorem 3.1. Assume that $\left(H_{1}\right)-\left(H_{3}\right)$ hold, then there is at least one solution of (2.1) on J.

Proof. For any $x(t) \in L_{2}^{\mathcal{F}}\left(J, R^{n}\right)$, define the operator $P$ by

$$
P x=z+\int_{0}^{t} f(s, x(s)) \mathrm{d} s+\int_{0}^{t} g(s, x(s)) \mathrm{d} B(s)+\sum_{0<\tau_{k}<t} I_{k}\left(x\left(\tau_{k}\right)\right) .
$$

By Lemma 2.3, we will first show that the operator $P$ is completely continuous.

Step 1. $P$ is continuous from $L_{2}^{\mp}\left(J, R^{n}\right)$ to $L_{2}^{\mp}\left(J, R^{n}\right)$. Let $x_{n}$ be a sequence such that $x_{n} \rightarrow x$ as $n \rightarrow \infty$ in $L_{2}^{\mp}\left(J, R^{n}\right)$, then

$$
\begin{aligned}
& \mathbb{E}\left\|P x_{n}-P x\right\|^{2} \\
& =\mathbb{E}\left\|\int_{0}^{t}\left(f\left(s, x_{n}\right)-f(s, x)\right) \mathrm{d} s+\int_{0}^{t}\left(g\left(s, x_{n}\right)-g(s, x)\right) \mathrm{d} B(s)+\sum_{0<\tau_{k}<t}\left(I_{k}\left(x_{n}\left(\tau_{k}\right)\right)-I_{k}\left(x\left(\tau_{k}\right)\right)\right)\right\|^{2} \\
& \leq 3 \mathbb{E}\left\|\int_{0}^{t}\left(f\left(s, x_{n}\right)-f(s, x)\right) \mathrm{d} s\right\|^{2}+3 \mathbb{E}\left\|\sum_{0<\tau_{k}<t}\left(I_{k}\left(x_{n}\left(\tau_{k}\right)\right)-I_{k}\left(x\left(\tau_{k}\right)\right)\right)\right\|^{2} \\
& \quad+3 \mathbb{E}\left\|\int_{0}^{t}\left(g\left(s, x_{n}\right)-g(s, x)\right) \mathrm{d} B(s)\right\|^{2} .
\end{aligned}
$$

By the Bunyakovskii inequality, one has

$$
\mathbb{E}\left\|\int_{0}^{t}\left(f\left(s, x_{n}\right)-f(s, x)\right) \mathrm{d} s\right\|^{2} \leq b \mathbb{E} \int_{0}^{t}\left\|f\left(s, x_{n}\right)-f(s, x)\right\|^{2} \mathrm{~d} s,
$$

and by the Itô isometry it follows that

$$
\mathbb{E}\left\|\int_{0}^{t}\left(g\left(s, x_{n}\right)-g(s, x)\right) \mathrm{d} B(s)\right\|^{2} \leq \mathbb{E} \int_{0}^{t}\left\|g\left(s, x_{n}\right)-g(s, x)\right\|^{2} \mathrm{~d} s .
$$


Hence, it can be obtained directly from (3.5)-(3.7) that

$$
\begin{aligned}
\mathbb{E}\left\|P x_{n}-P x\right\|^{2} \leq & 3 b \mathbb{E} \int_{0}^{t}\left\|f\left(s, x_{n}\right)-f(s, x)\right\|^{2} \mathrm{~d} s+3 \mathbb{E} \int_{0}^{t}\left\|g\left(s, x_{n}\right)-g(s, x)\right\|^{2} \mathrm{~d} s \\
& +3 m \sum_{0<\tau_{k}<t} \mathbb{E}\left\|I_{k}\left(x_{n}\left(\tau_{k}\right)\right)-I_{k}\left(x\left(\tau_{k}\right)\right)\right\|^{2} .
\end{aligned}
$$

Since $f$ is $L_{2}$-Carathéodory and $I_{k}$ are continuous functions, by the Lebesgue dominated convergence theorem, we see

$$
\begin{aligned}
\left\|P x_{n}-P x\right\|_{*}^{2} \leq & 3 b \mathbb{E} \int_{0}^{t}\left\|f\left(s, x_{n}\right)-f(s, x)\right\|^{2} \mathrm{~d} s+3 \mathbb{E} \int_{0}^{t}\left\|g\left(s, x_{n}\right)-g(s, x)\right\|^{2} \mathrm{~d} s \\
& +3 m \sum_{0<\tau_{k}<t} \mathbb{E}\left\|I_{k}\left(x_{n}\left(\tau_{k}\right)\right)-I_{k}\left(x\left(\tau_{k}\right)\right)\right\|^{2} \longrightarrow 0 \quad \text { as } n \longrightarrow \infty
\end{aligned}
$$

Step 2. $P$ maps bounded sets into bounded sets. That is enough to prove that there exists a positive constant $\theta$ such that for each $x(t) \in B_{q}=\left\{x \in L_{2}^{\mp}\left(J, R^{n}\right),\|x\| \leq q\right\}$ one has $\|P x\|_{*} \leq \theta$. By the definition in (3.4), for each $t \in J, x(t) \in B_{q}$,

$$
\begin{aligned}
\mathbb{E}\|P x\|^{2} & \leq 4 \mathbb{E}\|z\|^{2}+4 b \mathbb{E} \int_{0}^{t}\|f(s, x(s))\|^{2} \mathrm{~d} s+4 \mathbb{E} \int_{0}^{t}\|g(s, x(s))\|^{2} \mathrm{~d} s+4 m \sum_{0<\tau_{k}<t} c_{k}^{2} \\
& \leq 4 \mathbb{E}\|z\|^{2}+4 b \int_{0}^{t} h_{q}^{2}(s) \mathrm{d} s+4 \int_{0}^{t} h_{q}^{2}(s) \mathrm{d} s+4 m \sum_{0<\tau_{k}<t} c_{k}^{2} \\
& \leq 4 \mathbb{E}\|z\|^{2}+(4 b+4) \int_{0}^{t} h_{q}^{2}(s) \mathrm{d} s+4 m \sum_{0<\tau_{k}<t} c_{k}^{2}:=\theta .
\end{aligned}
$$

Step 3. P maps bounded sets into equicontinuous sets of $L_{2}^{\mp}\left(J, R^{n}\right)$.

Let $t_{1}, t_{2} \in J, t_{1}<t_{2}, x \in B_{q}$. Then, it yields

$$
\begin{aligned}
& \mathbb{E}\left\|(P x)\left(t_{2}\right)-(P x)\left(t_{1}\right)\right\|^{2} \\
& \quad \leq 3 m \sum_{t_{1} \leq t<t_{2}} \max _{k}\left\{c_{k}\right\}+3\left(t_{2}-t_{1}\right) \mathbb{E} \int_{t_{1}}^{t_{2}}\|f(s, x(s))\|^{2} \mathrm{~d} s+3 \mathbb{E} \int_{t_{1}}^{t_{2}}\|g(s, x(s))\|^{2} \mathrm{~d} s \\
& \quad \leq 3 m \sum_{t_{1} \leq t<t_{2}} \max \left\{c_{k}\right\}+3\left(t_{2}-t_{1}+1\right) \int_{t_{1}}^{t_{2}} h_{q}^{2}(s) \mathrm{d} s .
\end{aligned}
$$

As $t_{2} \rightarrow t_{1}$, the right-hand side of the inequality above tends to zero. The case $0<t_{2}<t_{1} \leq b$ can be considered in a similar manner.

From Ascoli-Arzela theorem and Steps 1 to 3, the operator $P$ is completely continuous. To complete the proof of the theorem, it suffices to prove the following step. 
Step 4. There exists a priori bound of the set

$$
F(P)=\left\{x \in L_{2}^{q}\left(J, R^{n}\right), x=\lambda P x \text { for some } \lambda \in(0,1)\right\} .
$$

Let $x(t) \in F(P)$, then $x(t)=\lambda P x(t)$ for some $0<\lambda<1$. Thus, for each $t \in J$,

$$
x(t)=\lambda\left(z+\int_{0}^{t} f(s, x(s)) \mathrm{d} s+\int_{0}^{t} g(s, x(s)) \mathrm{d} B(s)+\sum_{0<\tau_{k}<t} I_{k}\left(x\left(\tau_{k}\right)\right)\right),
$$

which implies that

$$
\mathbb{E}\|x(t)\|^{2} \leq 4 \mathbb{E}\|z\|^{2}+4 b \mathbb{E} \int_{0}^{t}\|f(s, x(s))\|^{2} \mathrm{~d} s+4 \mathbb{E} \int_{0}^{t}\|g(s, x(s))\|^{2} \mathrm{~d} s+4 m \sum_{0<\tau_{k}<t} c_{k}^{2} .
$$

Consider the function $\mu(t)$ defined by

$$
\mu(t)=\sup \left\{\mathbb{E}\|x(s)\|^{2}, 0 \leq s \leq t\right\}, \quad 0 \leq t \leq b .
$$

With the previous inequality, we have for $t \in[0, b]$

$$
\mu(t) \leq 4 \mathbb{E}\|z\|^{2}+(4 b+4) \int_{0}^{t} p^{2}(s) \psi^{2}(\mu(s)) d s+4 m \sum_{0<\tau_{k}<t} c_{k}^{2}
$$

Take the right-hand side of the inequality above as $v(t)$, then we get

$$
v(0)=c=4 \mathbb{E}\|z\|^{2}, \quad \mu(t) \leq v(t) \quad \forall t \in J,
$$

and, moreover,

$$
v^{\prime}(t)=(4 b+4) p^{2}(t) \psi^{2}(\mu(t)) \leq(4 b+4) p^{2}(t) \psi^{2}(v(t))
$$

or, equivalently, by $H_{3}$, for each $t \in J$,

$$
\int_{v(0)}^{v(t)} \frac{\mathrm{d} s}{\psi^{2}(s)} \leq \int_{0}^{t}(4 b+4) p^{2}(s) \mathrm{d} s \leq \int_{0}^{b}(4 b+4) p^{2}(s) \mathrm{d} s<\int_{c}^{\infty} \frac{\mathrm{d} s}{\psi^{2}(s)} .
$$

Hence, there exists a constant $k$ such that $v(t) \leq k, t \in J$, that is, $\mu(t)=\|x(t)\|_{*}^{2} \leq v(t) \leq k, t \in J$, which implies that $F(P)$ is bounded. From the Schaefer's fixed theorem, we deduce that $P$ has a fixed point $x(t)$ which is a solution to (2.1). The proof is completed.

In this part, in order to attain the uniqueness result of (2.1), we propose the following assumptions. 
$\left(A_{1}\right)$ There exists a square integrable function $l(t): J \rightarrow R_{+}$such that, for all $x, y \in$ $L_{2}^{\mp}\left(R^{n}\right)$ and $t \in J$,

$$
\|f(t, x)-f(t, y)\| \vee\|g(t, x)-g(t, y)\| \leq l(t)\|x-y\| .
$$

$\left(A_{2}\right)$ There exist positive constants $d_{k}, k=1,2, \ldots, m$ satisfying

$$
\left\|I_{k}(x)-I_{k}(y)\right\| \leq d_{k}\|x-y\|, \quad x, y \in L_{2}^{\mathcal{F}}\left(J, R^{n}\right)
$$

Theorem 3.2. Assume that $\left(A_{1}\right)-\left(A_{2}\right)$ hold, then there exists a unique solution to (2.1) if $\sum_{k=1}^{m} d_{k}<1$.

Proof. Let $P$ be an operator defined as in (3.4). To prove that $P$ is contractive, introduce the Bielecki-type norm on $L_{2}^{\mathcal{F}}\left(J, R^{n}\right)$ defined by

$$
\|x\|_{B}=\sup _{t \in J} e^{-\tau L(t)}\|x\|_{* \prime}
$$

where $L(t)=\int_{0}^{t} l^{2}(s) d s$ and $\tau$ is sufficiently large constant.

Let $x, y \in L_{2}^{\mp}\left(J, R^{n}\right)$, then we have that, for each $t \in J$,

$$
\begin{aligned}
& \|P x-P y\|_{*} \\
& \leq \int_{0}^{t}\|f(s, x)-f(s, y)\|_{*} \mathrm{~d} s+\left\|\int_{0}^{t}(g(s, x)-g(s, y)) \mathrm{d} B(s)\right\|_{*}+\sum_{0<\tau_{k}<t}\left\|I_{k}\left(x\left(\tau_{k}\right)\right)-I_{k}\left(y\left(\tau_{k}\right)\right)\right\|_{*} \\
& \leq\left(\int_{0}^{t} b l^{2}(s)\|x-y\|_{*}^{2} \mathrm{~d} s\right)^{1 / 2}+\left(\int_{0}^{t}\|g(t, x)-g(t, y)\|_{*}^{2} \mathrm{~d} s\right)^{1 / 2}+\sum_{k=1}^{m} d_{k}\|x-y\|_{*} \\
& =\left(\int_{0}^{t} b l^{2}(s) e^{2 \tau L(s)} e^{-2 \tau L(s)}\|x-y\|_{*}^{2} \mathrm{~d} s\right)^{1 / 2}+\left(\int_{0}^{t} l^{2}(s) e^{2 \tau L(s)} e^{-2 \tau L(s)}\|x-y\|_{*}^{2} \mathrm{~d} s\right)^{1 / 2} \\
& \quad+\sum_{k=1}^{m} d_{k} e^{\tau L(t)} e^{-\tau L(t)}\|x-y\|_{*} \\
& \leq\|x-y\|_{B}\left(\frac{1}{2 \tau} \int_{0}^{t} b\left(e^{2 \tau L(s)}\right)^{\prime} \mathrm{d} s\right)^{1 / 2}+\|x-y\|_{B}\left(\frac{1}{2 \tau} \int_{0}^{t}\left(e^{2 \tau L(s)}\right)^{\prime} \mathrm{d} s\right)^{1 / 2} \\
& \quad+\|x-y\|_{B} e^{\tau L(t)} \sum_{k=1}^{m} d_{k}
\end{aligned}
$$




$$
\begin{aligned}
& \leq\|x-y\|_{B} \frac{1}{\sqrt{2 \tau}}(\sqrt{b}+1) e^{\tau L(t)}+\sum_{k=1}^{m} d_{k} e^{\tau L(t)}\|x-y\|_{B} \\
& \leq e^{\tau L(t)}\left(\sqrt{\frac{1}{2 \tau}}(\sqrt{b}+1)+\sum_{k=1}^{m} d_{k}\right)\|x-y\|_{B} .
\end{aligned}
$$

Thus,

$$
e^{-\tau L(t)}\|P x-P y\|_{*} \leq\left(\sqrt{\frac{1}{2 \tau}}(\sqrt{b}+1)+\sum_{k=1}^{m} d_{k}\right)\|x-y\|_{B^{\prime}}
$$

therefore,

$$
\|P x-P y\|_{B} \leq\left(\sqrt{\frac{1}{2 \tau}}(\sqrt{b}+1)+\sum_{k=1}^{m} d_{k}\right)\|x-y\|_{B} .
$$

Hence, $P$ is a contraction and it has a unique fixed point which is a solution to (2.1). The proof is completed.

Remark 3.3. Note that the existence and uniqueness result in [7] can be inferred from Theorems 3.1 and 3.2 in case that $I_{k}=0$ in (2.1). Furthermore, if the systems in [9] and (2.1) are both reduced to the impulsive differential equations, then we can deduce the results on existence and uniqueness in [9] directly from Theorems 3.1 and 3.2. And, if the delay $=0$ in [2], then the conditions there can be inferred from Theorems 3.1 and 3.2 in case that $g=0$ in (2.1).

\section{Example}

Consider the following stochastic impulsive differential equation:

$$
\begin{aligned}
& \mathrm{d} x=(1+t) x \mathrm{~d} t+\left(t^{3}+1\right) x \mathrm{~d} B(t), \quad t \in[0,1] \backslash\left\{\frac{1}{2}\right\}, \\
& x\left(\frac{1}{2}^{+}\right)-x\left(\frac{1}{2}^{-}\right)=\frac{1}{4} \arctan \left(x\left(\frac{1}{2}^{-}\right)\right), \\
& x_{0}=0
\end{aligned}
$$

where $I_{1}(x)=(1 / 4) \arctan x$ with $\left\|I_{1}(x)\right\| \leq \pi / 8$ and

$$
\left\|I_{1}(x)-I_{1}(y)\right\| \leq \frac{1}{4}\|x-y\| \quad \forall x, y \in L_{2}^{\mp}\left(J, R^{n}\right) .
$$


Assume that $p(t)=1+t+t^{3}$ and $\psi(x)=x$, then it can be easily verified that the conditions $\left(H_{1}\right)-\left(H_{3}\right)$ are satisfied, and by, Theorem 3.1, (4.1) has at least one solution.

For all $x, y \in L_{2}^{\mp}\left(J, R^{n}\right)$ and $t \in[0,1]$, set $l(t)=1+t+t^{3}$, then

$$
\|f(t, x)-f(t, y)\| \vee\|g(t, x)-g(t, y)\| \leq l(t)\|x-y\| .
$$

As stated in (4.2), $d_{k}=1 / 4<1$; hence, (4.1) has a unique solution by Theorem 3.2.

As demonstrated in the example, if $f$ or $g$ takes the form such as $p(t) x^{2}$, then inequality (3.3) in hypothesis $\left(H_{3}\right)$ cannot hold. Based on this observation, in the subsequent research, some useful tools and conditions such as locally Lipschitz condition will be considered to relax restrictions on $f$ and $g$.

\section{Conclusion}

By using Schaefer's fixed theorem, this paper has obtained sufficient conditions of existence and uniqueness of mild solutions to general nonlinear stochastic impulsive differential equations. In addition, some known results about systems without delay or impulse can be considered as special cases of the main theorems.

\section{Acknowledgment}

This work is supported by the NSF of China under Grants 60874027,61174039, the Fundamental Research Funds for the Central Universities of China, and by Youth Science Foundation of Luoyang Normal University (2010-qnij-002).

\section{References}

[1] V. Lakshmikantham, D. D. Baĭnov, and P. S. Simeonov, Theory of Impulsive Differential Equations, vol. 6, World Scientific Publishing., Teaneck, NJ, USA, 1989.

[2] A. Ouahab, "Local and global existence and uniqueness results for impulsive functional differential equations with multiple delay," Journal of Mathematical Analysis and Applications, vol. 323, no. 1, pp. 456-472, 2006.

[3] M. Perestyuk and P. Feketa, "Invariant sets of impulsive differential equations with particularities in w-limit set," Abstract and Applied Analysis, vol. 2011, Article ID 970469, 14 pages, 2011.

[4] S. Peng and L. Yang, "Global exponential stability of impulsive functional differential equations via Razumikhin technique," Abstract and Applied Analysis, vol. 2010, Article ID 987372, 11 pages, 2010.

[5] B. A. Ton, "Exact controllability for a nonlinear stochastic wave equation," Abstract and Applied Analysis, vol. 2006, Article ID 74264, 14 pages, 2006.

[6] Z. Wang, Y. Wang, and Y. Liu, "Global synchronization for discrete-time stochastic complex networks with randomly occurred nonlinearities and mixed time delays," IEEE Transactions on Neural Networks, vol. 21, no. 1, pp. 11-25, 2010.

[7] B. Oksendal, Stochastic Differential Equations, Springer, Berlin, Germany, 5th edition, 2002.

[8] X. R. Mao, Stochastic Differential Equations and Applications, Horwood, Chichester, UK, 1997.

[9] H. Bao and J. Cao, "Existence and uniqueness of solutions to neutral stochastic functional differential equations with infinite delay," Applied Mathematics and Computation, vol. 215, no. 5, pp. 1732-1743, 2009.

[10] B. Liu, X. Liu, and X. Liao, "Existence and uniqueness and stability of solutions for stochastic impulsive systems," Journal of Systems Science E Complexity, vol. 20, no. 1, pp. 149-158, 2007.

[11] L. Hu and Y. Ren, "Existence results for impulsive neutral stochastic functional integro-differential equations with infinite delays," Acta Applicandae Mathematicae, vol. 111, no. 3, pp. 303-317, 2010. 
[12] M. S. Alwan, X. Liu, and W.-C. Xie, "Existence, continuation, and uniqueness problems of stochastic impulsive systems with time delay," Journal of the Franklin Institute, vol. 347, no. 7, pp. 1317-1333, 2010.

[13] H. Zhang, Z.-H. Guan, and G. Feng, "Reliable dissipative control for stochastic impulsive systems," Automatica, vol. 44, no. 4, pp. 1004-1010, 2008.

[14] Z. M. Liu and J. Peng, "p-moment stability of stochastic nonlinear delay systems with impulsive jump and Markovian switching," Stochastic Analysis and Applications, vol. 27, no. 5, pp. 911-923, 2009. 


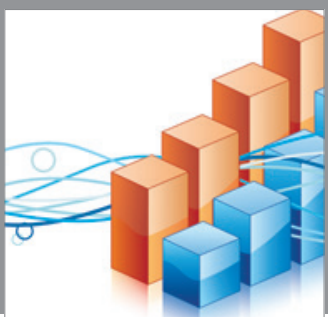

Advances in

Operations Research

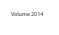

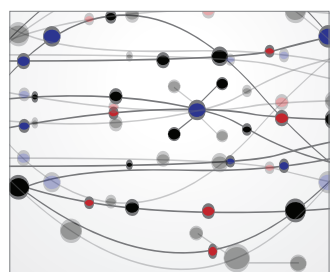

\section{The Scientific} World Journal
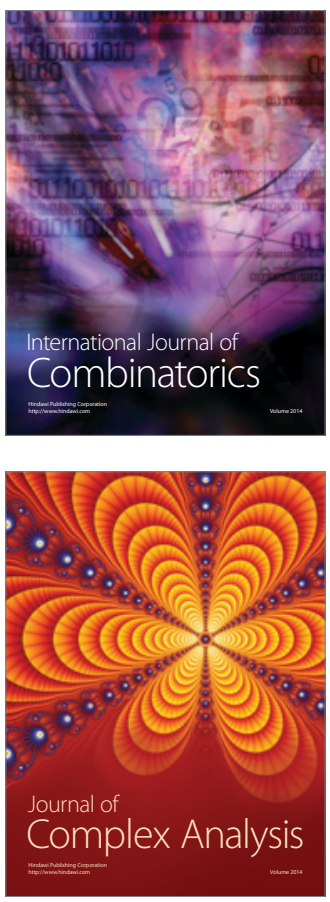

International Journal of

Mathematics and

Mathematical

Sciences
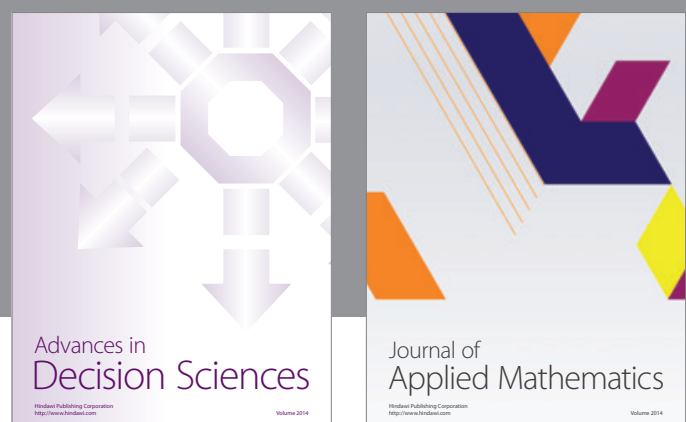

Journal of

Applied Mathematics
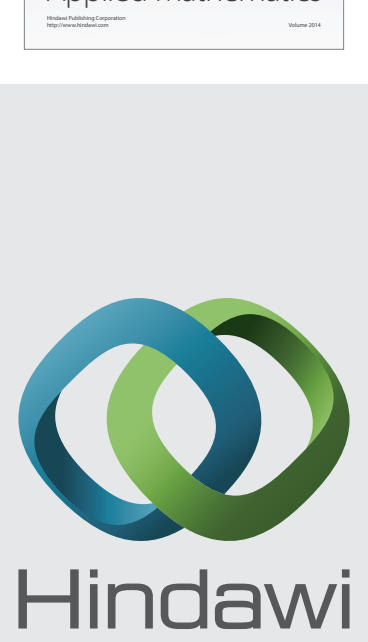

Submit your manuscripts at http://www.hindawi.com
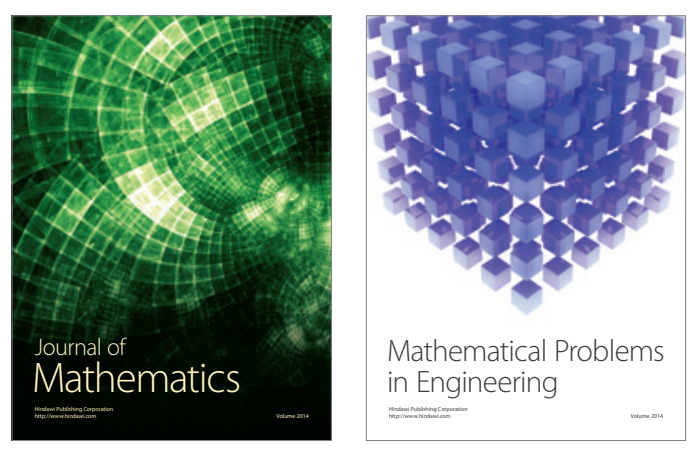

Mathematical Problems in Engineering
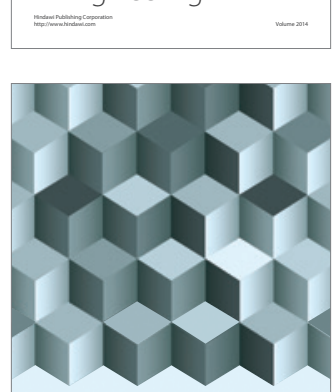

Journal of

Function Spaces
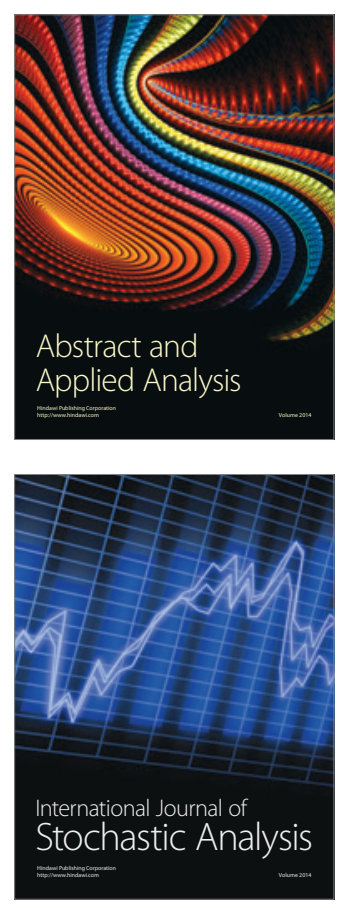

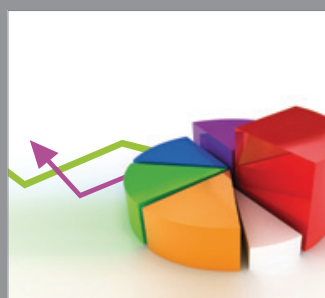

ournal of

Probability and Statistics

Promensencen
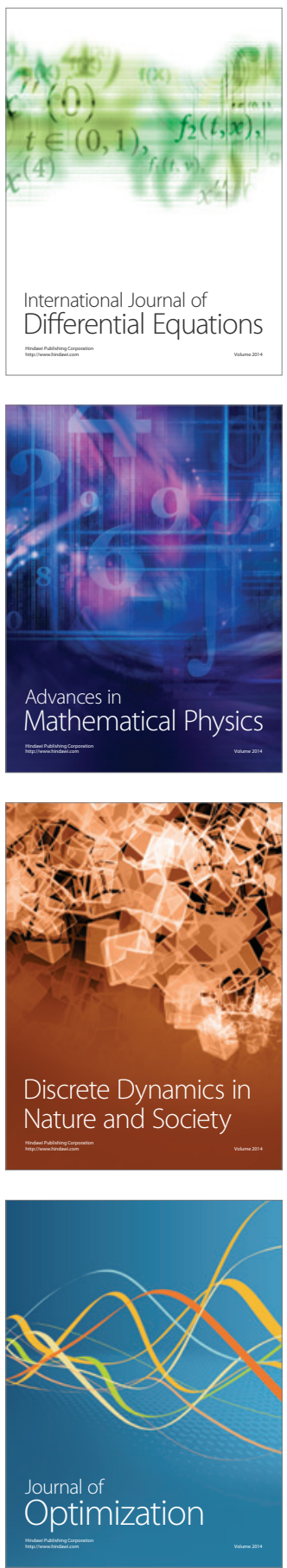